OS10 (2) - 5 (OS10W0102)

\title{
Standard Mechanical Testings for Solders - JSMS Recommendation -
}

\author{
Masao Sakane, Dept. of Mechanical Engineering, Ritsumeikan Univ., Kusatsu, Japan \\ Haruo Nose, Dept. of Mechanical Engineering, Osaka Sangyo Univ., Daito, Japan \\ Makoto Kitano, Mechanical Engineering Research Laboratory, Hitachi, Ltd., Tsuchiura, Japan \\ Hiroyuki Takahashi, Corporate Research \& Development Center, Toshiba Corporation, Kawasaki, Japan \\ Minoru Mukai, Corporate Research \& Development Center, Toshiba Corporation, Kawasaki, Japan \\ Yutaka Tsukada, IBM Japan Ltd., Yasu, Japan
}

This study describes the standard mechanical testing methods for solders. The mechanical testing methods discussed are for tensile, low cycle fatigue, creep and creep-fatigue. These standard testing methods were recommended by the subcommittee on solder strength in the Society of Materials Science, Japan. The former two standards were already published in 2001 and the latter two standards are under discussion for publication. They will be published in 2003. Tensile and low cycle fatigue database was also published. The database has the tensile and low cycle data of $\mathrm{Sn}-37 \mathrm{~Pb}$ and $\mathrm{Sn}-3.5 \mathrm{Ag}$ at $313 \mathrm{~K}, 353 \mathrm{~K}$ and $398 \mathrm{~K}$. The main points of the standards are as follows.

The standards recommend testing methods for obtaining the stable data by minimizing the scatter. They proposed the casting method, specimen geometry, testing procedure and the data be reported in detail. For the casting method, the casting temperature recommended was $100 \mathrm{~K}$ above the melting points of solders. Materials and dimensions of mould for casting were also recommended to have a stable microstructure of solders.

In the tensile testing standard, a strain rate of $2.0 \% / \mathrm{s}$ was recommended to exclude the creep deformation from stress-strain curves of solders, since solders significantly creep even at room temperature. The temperature of specimens should be controlled and monitored during tensile testing.

A new extensometer attaching method was proposed to avoid cracking at the pressing place of extensometer rods in the low cycle fatigue standard. Shape and dimensions of standard specimens were proposed. Strain wave should be a full reversed triangular and the strain rate $0.1 \% / \mathrm{s}$. This strain rate is slower than that used in tensile testing, but it was recommended to avoid the difficulty of carrying out experiments with much faster strain rate. Another reason of employing this strain rate is a small difference of fatigue life in symmetrical strain waves independent strain rate.

Creep and creep-fatigue standards are finalizing stage for publication. Specimen geometry and testing devices for creep testing will be proposed as the creep testing standard. An approximation method of steady creep curves will be also proposed. Accurate creep-fatigue life prediction methods will be proposed. Finite conclusions will be obtained until the ATEM conference, so that rigid standard testing methods for creep and creep-fatigue testing will be presented at the conference. 